\title{
Combining Modulated Techniques for the Analysis of Photosensitive Devices
}

\author{
Agustin O. Alvarez,* Sandheep Ravishankar, and Francisco Fabregat-Santiago*
}

Small-perturbation techniques such as impedance spectroscopy (IS), intensity-modulated photocurrent spectroscopy (IMPS), and intensity-modulated photovoltage spectroscopy (IMVS) are useful tools to characterize and model photovoltaic and photoelectrochemical devices. While the analysis of the impedance spectra is generally carried out using an equivalent circuit, the intensity-modulated spectroscopies are often analyzed through the measured characteristic response times. This makes the correlation between the two methods of analysis generally unclear. In this work, by taking into consideration the absorptance and separation efficiency, a unified theoretical framework and a procedure to combine the spectral analysis of the three techniques are proposed. Such a joint analysis of IS, IMPS, and IMVS spectra greatly reduces the sample space of possible equivalent circuits to model the device and allows obtaining parameters with high reliability. This theoretical approach is applied in the characterization of a silicon photodiode to demonstrate the validity of this methodology, which shows great potential to improve the quality of analysis of spectra obtained from frequency domain small-perturbation methods.

\section{Introduction}

Intensity-modulated photocurrent spectroscopy (IMPS), intensity-modulated photovoltage spectroscopy (IMVS), and impedance spectroscopy (IS) are powerful techniques to characterize electrochemical and solid-state light-to-energy conversion devices under operational conditions. ${ }^{[1,2]}$ IMPS was extensively developed by Peter and co-workers ${ }^{[3,4]}$ mainly in the characterization of carrier transport processes, ${ }^{[5-8]}$ while IMVS has been mostly used in the study of recombination processes, though both tech-

\author{
A. O. Alvarez, F. Fabregat-Santiago \\ Institute of Advanced Materials \\ Universitat Jaume I \\ Castelló de la Plana 12006, Spain \\ E-mail: agalvare@uji.es; fabresan@uji.es \\ S. Ravishankar \\ IEK-5 Photovoltaik \\ Forschungzentrum Jülich \\ 52425 Jülich, Germany
}

The ORCID identification number(s) for the author(s) of this article can be found under https://doi.org/10.1002/smtd.202100661.

(C) 2021 The Authors. Small Methods published by Wiley-VCH GmbH. This is an open access article under the terms of the Creative Commons Attribution-NonCommercial-NoDerivs License, which permits use and distribution in any medium, provided the original work is properly cited, the use is non-commercial and no modifications or adaptations are made.

DOI: 10.1002/smtd.202100661 niques involve optical perturbations. ${ }^{[9,10]}$ IS has been used to analyze transport and recombination processes together through the response of the device to an electrical perturbation. ${ }^{[11,12]}$

IMPS and IMVS techniques have been widely applied to study dye-sensitized solar cells, ${ }^{[13,14]}$ photoelectrodes for water oxidation, ${ }^{[15-17]}$ and recently are gaining attention in the field of perovskite solar cells (PSCs). ${ }^{[18,19]}$ The analysis of the data obtained with these techniques is generally made through the characteristic time constants. ${ }^{[20-22]}$ While such an analysis is very useful in certain cases, this strategy limits the amount of information that can be obtained from these techniques. With regard to IS, experimental data are usually analyzed by modeling the internal electrochemical processes of the device using an equivalent circuit model (EC), ${ }^{[23]}$ built from passive electrical elements such as resistances, capacitances, and inductances. ${ }^{[24-26]}$ These parameters in turn can provide key information regarding the nature of the operation of the device and its limitations. However, the choice of the correct EC could be a difficult task, because several ECs can reproduce the same experimental spectra. Therefore, there exists a need to combine the analysis methods of these three techniques to overcome their individual limitations and extract the maximum knowledge from the experimental data.

Before discussing how these techniques are related, we present a scheme of a photosensitive device showing the basic light to electricity conversion mechanism in Figure 1. In these systems, when a photon flux $(\phi)$ reaches the device, part of these photons can be absorbed, generating electronhole pairs (represented by absorbed current, $j_{\mathrm{a}}$ ). The pairs that do not recombine and are successfully separated, provide the free-photogenerated current $\left(j_{\mathrm{ph}}\right)$ in the light absorber material. Part of this current is lost as a recombination current $\left(j_{\text {rec }}\right)$, and the remaining current $\left(j_{\mathrm{e}}=j_{\mathrm{ph}}-j_{\mathrm{rec}}\right)$ is extracted at the external contacts of the device. $j_{\text {rec }}$, and thus $j_{\mathrm{e}}$, are functions of the external voltage $\left(V_{\mathrm{e}}\right)$.

In Figure 2, the basic operation of IS, IMPS, and IMVS is shown. Figure $2 \mathrm{~b}-\mathrm{d}$ shows the excitation and measurement signals involved in IS, IMPS, and IMVS, respectively. Note that all variables with a tilde $(\tilde{)})$ indicate $\mathrm{AC}$ perturbations, while variables with overbars $(-)$ represent steady-state signals. Thus, the steady-state at which the measurements are performed is defined by $\bar{j}_{\phi}, \bar{V}_{\mathrm{e}}$, and $\bar{j}_{\mathrm{e}}$. As shown in Figure $2 \mathrm{~b}$, for IS (in potentiostatic mode), an AC small-perturbation in voltage 


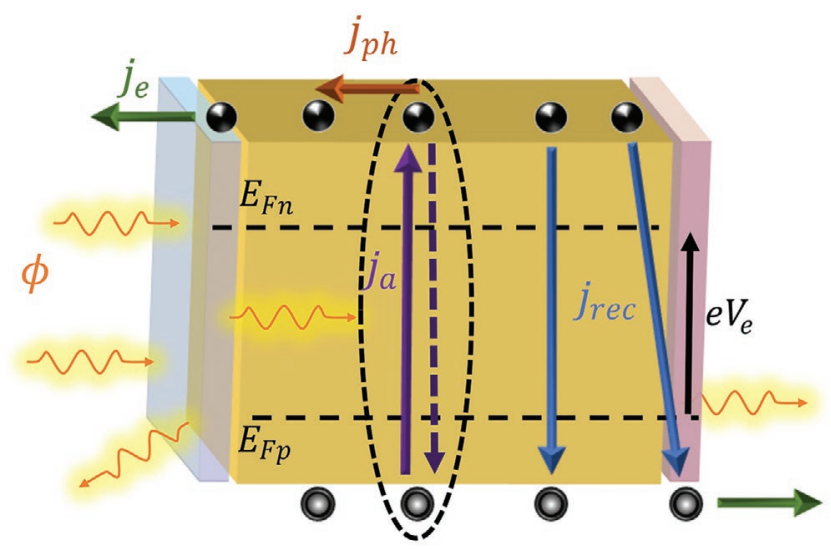

Figure 1. Scheme of the light to electricity conversion mechanism in a photovoltaic device. $\phi$ is a photon flux reaching the device. $j_{a}, j_{\text {ph }}, j_{\text {rec }}$, and $j_{\text {e }}$ are the absorbed, free-photogenerated, recombination, and extracted currents, respectively. $E_{\mathrm{Fn}}$ and $E_{\mathrm{Fp}}$ are the Fermi levels of electrons and holes, respectively, $e$ is the elementary charge and $V_{\mathrm{e}}$ is the external voltage.

$\left(\widetilde{V}_{\mathrm{e}}\right)$ is applied and the corresponding AC external current $\left(\tilde{j}_{\mathrm{e}}\right)$ is measured. The IS transfer function is then

$Z=\frac{\widetilde{V}_{\mathrm{e}}}{\tilde{j}_{\mathrm{e}}}$

Similarly, for IMPS and IMVS, a perturbation of incident photon current density $\left(\tilde{j}_{\phi}=e \tilde{\phi}\right)$ is applied and $\tilde{j}_{\mathrm{e}}$ and $\widetilde{V}_{\mathrm{e}}$ are measured, respectively. If we define the generated photocurrent as negative and, consequently, the photovoltage as positive, then the IMPS and IMVS transfer functions are

$$
\begin{aligned}
& Q=-\frac{\tilde{j}_{\mathrm{e}}}{\tilde{j}_{\phi}} \\
& W=\frac{\widetilde{V}_{\mathrm{e}}}{\tilde{j}_{\phi}}
\end{aligned}
$$

Note that if we use the opposite notation (also very common), in which the generated photocurrent is positive and the photovoltage negative, ${ }^{[27,28]}$ then the signs of IMPS and IMVS change yielding $Q=\tilde{j}_{\mathrm{e}} / \tilde{j}_{\phi}$ and $W=-\widetilde{V}_{\mathrm{e}} / \tilde{j}_{\phi}$, while the definition of $Z$ remains unchanged.

As shown in Figure 2, these three techniques are closely related. The basic relationship between the transfer functions, derived step by step in Section S1.1 in the Supporting Information, is

$$
Z(\omega)=\frac{W(\omega)}{Q(\omega)}
$$

This relation was confirmed from measurements on different systems such as dye-sensitized solar cells, ${ }^{\left[{ }^{[2]}\right]}$ water splitting, ${ }^{[2]}$ and PSCs. ${ }^{[27]}$ On the other hand, Almora et al. recently showed some particular cases where this relation might not be entirely valid. ${ }^{[30,31]}$ Therefore, the validity of Equation (4) is unquestionable only after verification. It is worth highlighting the fact that to be in the regime where Equation (4) is valid, strict conditions must be fulfilled: i) The device is stable enough to ensure that the three techniques are performed in
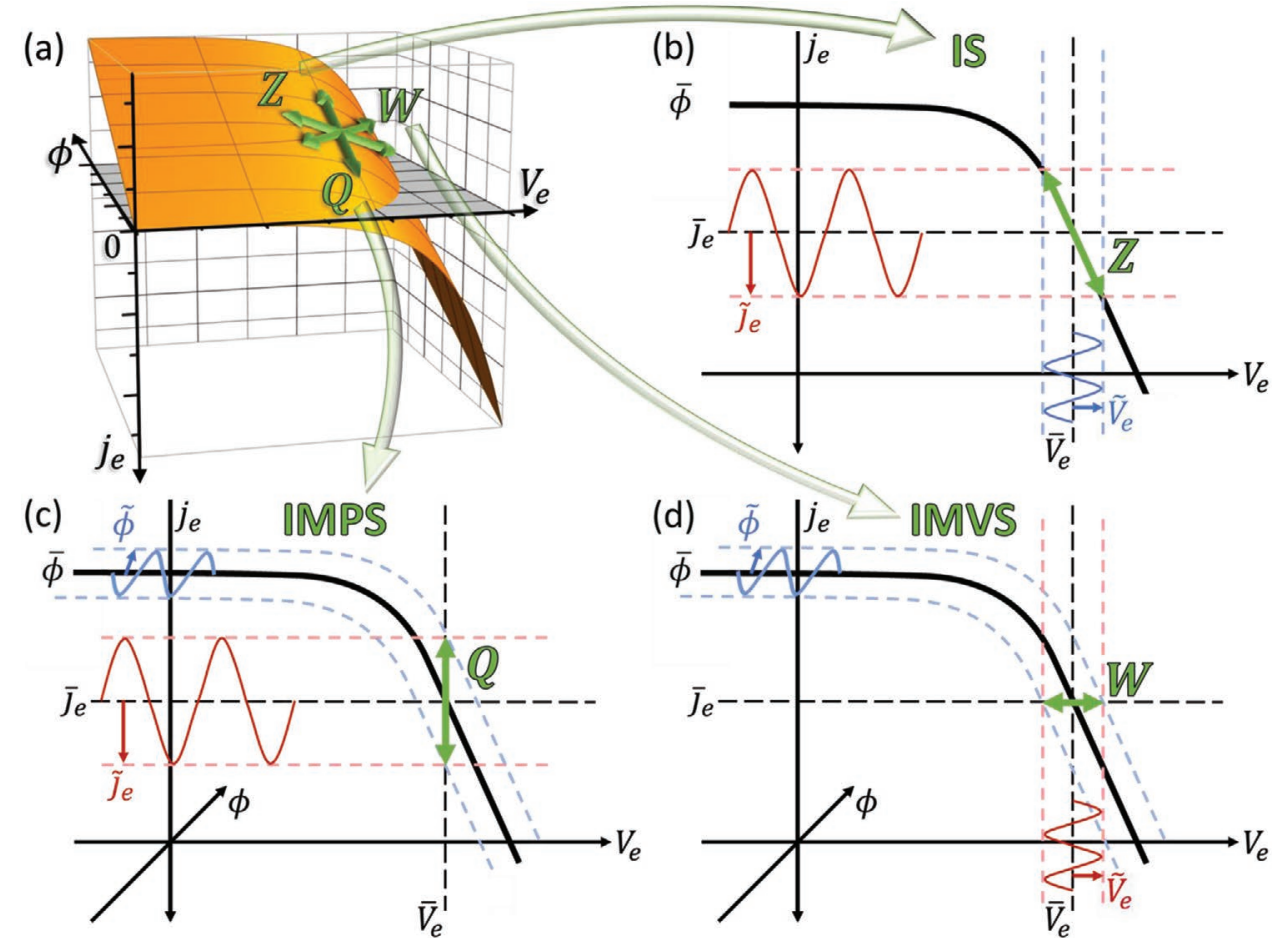

Figure 2. a) Characteristic current-voltage-photon flux $\left(j_{e}-V_{e}-\phi\right)$ surface for a device as the one shown in Figure 1. b-d) Represent the connection between the current-voltage curve and the transfer functions of IS (Z), IMPS (Q), and IMVS (W), respectively. The tilde represents AC signals on top of the DC values (represented by the overbar). The AC perturbation is shown in blue, the modulated response is shown in red, and the corresponding transfer function is shown in green. The slope of the surface in the $\phi, V_{\mathrm{e}}$, and $j_{\mathrm{e}}$ planes provide the DC limit of these transfer functions. 
the same steady-state condition ${ }^{[32,33]}$ and ii) the different perturbations applied are small enough to ensure linearity of the system response. An additional point to take into consideration is that different Fermi-level distributions in the material during measurement of each spectrum could affect this relation.

Other relations among these techniques have been previously made. Klotz et al. combined the three techniques and analyzed them by means of a distribution of relaxation times. ${ }^{[2]}$ This procedure can be particularly useful to separate polarization processes, but its implementation is not straightforward. Bertoluzzi and Bisquert theoretically proposed for the first time that a common EC linking the three small-perturbation techniques should describe the same physical mechanisms occurring for a device. ${ }^{[1]}$ Applying this theory and assuming perfect free-carrier photogeneration $\left(j_{\phi}=j_{\mathrm{ph}}\right)$, Ravishankar et al. proposed an EC to describe the frequency-domain, small-perturbation response of PSCs. ${ }^{[34]}$ In this last work, the authors measured IS and IMPS, reproducing both measured spectra with the EC proposed. However, some discrepancies were observed in the parameters obtained from the fitting of experimental spectra with the two techniques.

In the present work, we advance the state-of-the-art analysis by taking into account the contribution of the absorptance and the separation efficiency to accurately correlate the IMPS and IMVS responses with the IS response and their correspondences in the fitting of experimental spectra. We first introduce a general equivalent circuit (GEC) composed of generic impedance elements. Then, we derive the corresponding expressions for the IS, IMPS, and IMVS transfer functions of this GEC and the inter-relation among these techniques, which allows combining and complementing the information obtained with the three techniques in a unified analysis procedure. These results may be applied to many different systems by just adapting the impedances in the GEC to the specific combination of elements needed to describe each device. Finally, to illustrate the validity and capacity of the method, we characterized a silicon photodiode with our developed theoretical framework. We started by combining the three techniques to narrow down the large number of potential equivalent circuits available to analyze the IS data using a selection method, identifying an appropriate equivalent circuit that models all three experimental spectra. Such an analysis leads to a consistent set of output parameters that combines the information obtained from each individual measurement technique, allowing for a more holistic analysis of the optoelectronic properties of the device.

\section{Results and Discussion}

\subsection{Theory: General Equivalent Circuit}

\subsubsection{Theoretical Framework}

The photovoltaic external quantum efficiency $\left(\mathrm{EQE}_{\mathrm{PV}}\right)$ relates the flux of collected electrons per incident photon $\left(j_{\mathrm{e}}=\mathrm{EQE}_{\mathrm{PV}} j_{\phi}\right)$ and can be expressed as the product of three terms

$E Q E_{P V}=a \eta_{s e p} \eta_{c o l}$

where $a$ is the absorptance, also called light-harvesting efficiency (LHE or $\eta_{\mathrm{LHE}}$ ), defined by the fraction of incident photons that are effectively absorbed $\left(j_{\mathrm{a}}=a j_{\phi}\right.$, see Figure 1 ). $\eta_{\text {sep }}$ is the efficiency of charge separation from the generated electron-hole pairs to free-photogenerated carriers $\left(j_{\text {ph }}=\eta_{\text {sep }} j_{\mathrm{a}}\right) \cdot j_{\text {ph }}$ represents the current provided by the current generator in the equivalent circuit of the device. Finally, $\eta_{\text {col }}$ is the collection efficiency that determines the fraction of free-photogenerated carriers that are extracted at the contacts $\left(j_{\mathrm{e}}=\eta_{\mathrm{col}} j_{\mathrm{ph}}\right) \cdot{ }^{[35]}$ The photovoltaic internal quantum efficiency $\left(\mathrm{IQE}_{\mathrm{PV}}\right)$ is the ratio between collected electrons or holes and absorbed photons $\left(j_{\mathrm{e}}=\mathrm{IQE} \mathrm{EV}_{\mathrm{PV}} j_{\mathrm{a}}\right)$, so

$I Q E_{P V}=\eta_{\text {sep }} \quad \eta_{\text {col }}$

Similarly, the ratio of incident photons and free-photogenerated carriers can be expressed as the absorptance and separation efficiency

$\eta_{\mathrm{ass}}=a \eta_{\text {sep }}$

which implies $j_{\mathrm{ph}}=\eta_{\mathrm{a} \cdot \mathrm{s}} j_{\phi}$. Assuming that $\eta_{\mathrm{a} \cdot \mathrm{s}}$ is independent of the light intensity, the modulated photon flux and the freephotogenerated carriers are related by the relationship

$\tilde{j}_{p h}=\eta_{\mathrm{a} \cdot \mathrm{s}} \tilde{j}_{\phi}$

In homogenous semiconductors with low exciton binding energies, such as crystalline silicon (c-Si) or most inorganic thin films, $\eta_{\text {sep }} \approx 1$ could be a good approximation. ${ }^{[35,36]}$ In such cases, we can simplify $\eta_{\mathrm{a} \cdot \mathrm{s}} \approx a$, a parameter that can be measured directly. However, this is not the general case, especially in photoelectrochemical systems or solar cells that are in an optimization stage. Therefore, we will use $\eta_{\text {a.s }}$ as a single parameter to describe the efficiency of absorption and separation.

\subsubsection{Transfer Functions}

Using Equation (8), the IMPS and IMVS transfer functions in Equations (2) and (3) can be rewritten as

$Q=-\eta_{\mathrm{a} \cdot \mathrm{s}} \frac{\tilde{j}_{\mathrm{e}}}{\tilde{j}_{p h}}$

$\mathrm{W}=\eta_{\mathrm{a} \cdot \mathrm{s}} \frac{\widetilde{V}_{\mathrm{e}}}{\tilde{j}_{p h}}$

Equations (9) and (10) have an important consequence as they allow combining and comparing light-modulated methods with voltage or current-modulated methods. Without proper normalization of the IMPS and IMVS transfer functions using the absorption and separation efficiency, any comparison of calculated parameters from these methods will be skewed from those measured by IS. Therefore, in systems where the separation efficiency is 1 , the absorptance, which is a straightforward optical quantity that can be easily determined, is essential for an accurate normalization of the transfer functions. With these relations, we can now introduce an electrical model of the device. To be as general as possible, we assume 


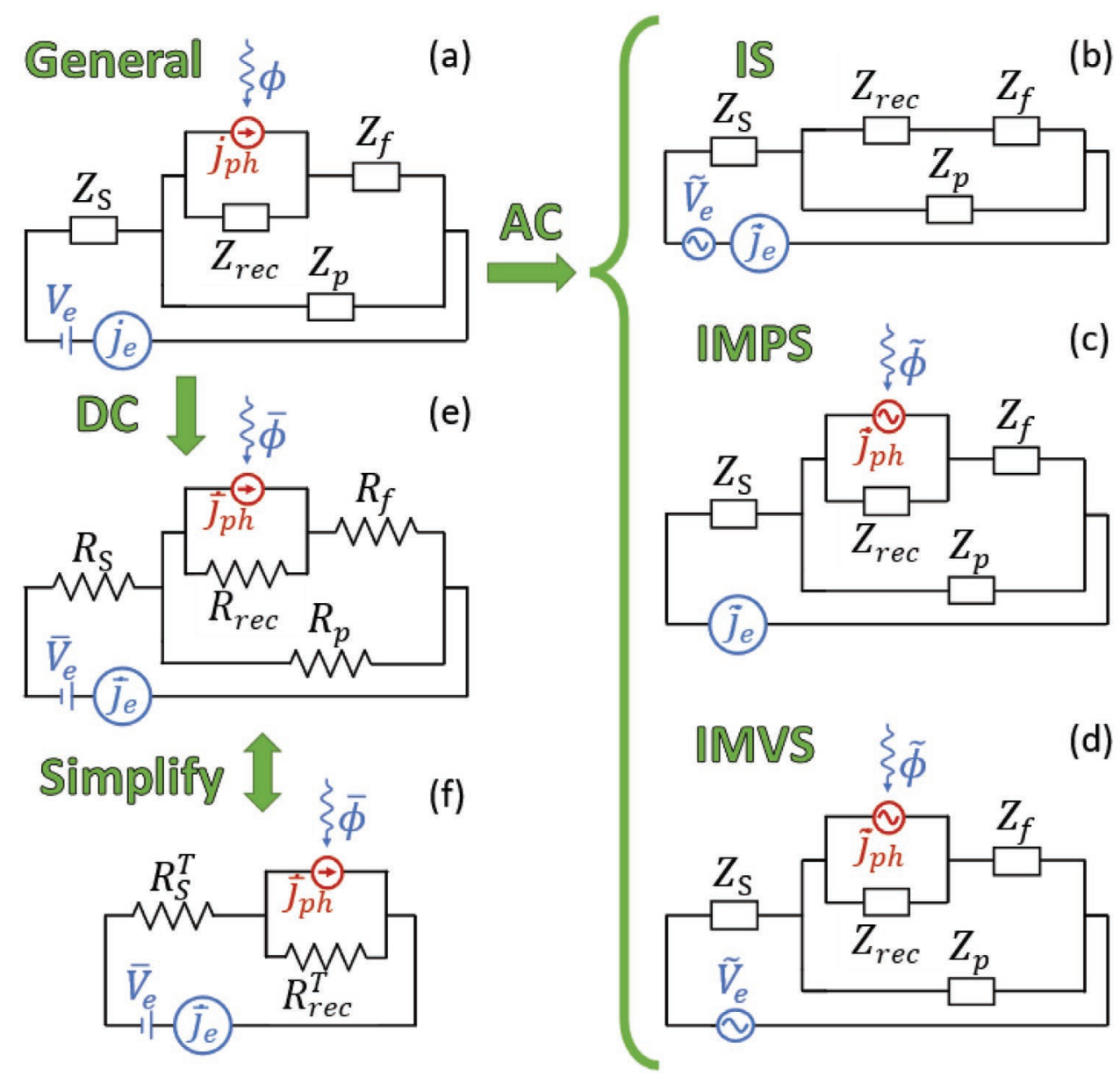

Figure 3. a) General equivalent circuit, with the simplifications to b) IS, c) IMPS, d) IMVS, and e,f) steady-state condition. $\phi$ is a photon flux reaching the device. $j_{\mathrm{ph}}$ and $j_{\mathrm{e}}$ are the free-photogenerated and extracted currents. $Z_{\mathrm{s}}, Z_{\mathrm{rec}}, Z_{\mathrm{p}}$, and $Z_{\mathrm{f}}$ are the general impedances and $V_{\mathrm{e}}$ is the external voltage. The tilde and overbar represent the AC and DC signals, respectively. The directly measurable variables are represented in blue while the free-photogenerated current is in red. The "total variables" in (f) are electrically equivalent to those they substitute in (e), see also Figure S1 in the Supporting Information.

the GEC model shown in Figure 3a. This model is made up of four general impedances $\left(Z_{\mathrm{S}}, Z_{\text {rec }}, Z_{\mathrm{p}}\right.$, and $\left.Z_{\mathrm{f}}\right)$. The measurable variables $\left(\phi, V_{\mathrm{e}}\right.$, and $\left.j_{\mathrm{e}}\right)$ are represented in blue while the free-photogenerated current $\left(j_{\mathrm{ph}}\right)$, which is directly unmeasurable, is in red. This GEC may be adapted to represent fundamental processes such as charge transport, ${ }^{[1]}$ traps, ${ }^{[37]}$ geometric and chemical ${ }^{[38]}$ capacitances, etc., depending on the studied system.

The transfer function of impedance spectroscopy for the equivalent circuit in Figure $3 \mathrm{~b}$ may be obtained by considering a small perturbation of voltage (or current) on the GEC in Figure 3a and applying the superposition theorem and Kirchhoff's laws, which yields

$Z=Z_{\mathrm{s}}+\left(\frac{1}{Z_{\mathrm{p}}}+\frac{1}{Z_{\text {rec }}+Z_{\mathrm{f}}}\right)^{-1}$

Similarly, the transfer function for IMPS (Figure 3c) is

$Q=\frac{\eta_{\mathrm{a} \cdot \mathrm{s}}}{Z_{\mathrm{s}}} \frac{Z_{\text {rec }}}{Z_{r e c}+Z_{\mathrm{f}}}\left(\frac{1}{Z_{\mathrm{s}}}+\frac{1}{Z_{\mathrm{p}}}+\frac{1}{Z_{r e c}+Z_{\mathrm{f}}}\right)^{-1}$ and the IMVS transfer function (Figure 3d) is

$$
\mathrm{W}=\eta_{\mathrm{a} \cdot \mathrm{s}} \frac{Z_{\text {rec }}}{Z_{\text {rec }}+Z_{\mathrm{f}}}\left(\frac{1}{Z_{\mathrm{p}}}+\frac{1}{Z_{\text {rec }}+Z_{\mathrm{f}}}\right)^{-1}
$$

Alternative expressions and combinations of Equations (11)-(13) are shown in Section S1.2 of the Supporting Information. The low-frequency limit is also discussed in Section S1.3 of the Supporting Information, where it is shown that the GEC is reduced to Figure $3 \mathrm{e}$ and can be simplified to the EC in Figure $3 f$ by defining the "total" equivalent series $\left(R_{\mathrm{S}}^{\mathrm{T}}\right)$ and recombination $\left(R_{\text {rec }}^{\mathrm{T}}\right)$ resistances. Combining Equations (12) and (13) through Equation (4), Equation (11) is obtained again. Whenever Equation (4) is valid, all the information that can be extracted from the three techniques is contained in the combination of any two of them.

We will now explore a simplified case of the GEC with special implications that will be applied in the experimental part. 


\subsubsection{Case $Z_{f}=0$ (or $Z_{f} \ll Z_{\text {rec }}$ )}

This case is very illustrative for the simplicity of the resulting equations. The most important consequence of considering $Z_{\mathrm{f}}=0$ is that $Z_{\text {rec }}$ and $Z_{\mathrm{p}}$ may not be dissociated anymore and the two branches in Figure 3 reduce to a single "total recombination impedance"

$Z_{r e c}^{\mathrm{T}}=\left(\frac{1}{Z_{\mathrm{p}}}+\frac{1}{Z_{\text {rec }}}\right)^{-1}$

The IS, IMPS, and IMVS transfer functions in Equations (11)-(13) reduce to

$Z=Z_{\mathrm{S}}+Z_{\text {rec }}^{\mathrm{T}}$

$Q=\frac{\eta_{\mathrm{a} \cdot \mathrm{s}}}{Z_{\mathrm{s}}}\left(\frac{1}{Z_{\mathrm{s}}}+\frac{1}{Z_{\text {rec }}^{\mathrm{T}}}\right)^{-1}$

$\mathrm{W}=\eta_{\mathrm{a}: \mathrm{S}} Z_{\mathrm{rec}}^{\mathrm{T}}$

Note that the GEC is reduced to only two impedances $Z_{S}$ and $Z_{\text {rec }}^{\mathrm{T}}$, and they can be separated using any couple of the experimental techniques plus $\eta_{\text {a.s }}$ without making any further assumptions, through

$Z_{\text {rec }}^{\mathrm{T}}=\frac{W}{\eta_{\mathrm{a} \cdot \mathrm{s}}}=\frac{Z Q}{\eta_{\mathrm{a} \cdot \mathrm{s}}}$

and

$Z_{\mathrm{s}}=W\left(\frac{1}{Q}-\frac{1}{\eta_{\mathrm{a} \cdot \mathrm{s}}}\right)=Z\left(1-\frac{Q}{\eta_{\mathrm{a} \cdot \mathrm{s}}}\right)=Z-\frac{W}{\eta_{\mathrm{a} \cdot \mathrm{s}}}$

These calculations represent clear progress for the definition of an accurate EC of the device. Therefore, the serial contributions of the impedance, such as contact or transport impedances, can be separated from those which are in parallel to the absorber and charge generator, such as recombination and some polarization processes. This is a key aspect to model and understand the operation and limitations of many optoelectronic and photovoltaic devices. ${ }^{[1,39]}$

\subsection{Experimental Demonstration: Silicon Photodiode}

To illustrate our theoretical development with experimental results, we measured the IS, IMPS, and IMVS spectra of a commercial silicon photodiode (Thorlabs FDS100), see black dots in Figure 4. Silicon photodiodes are well-known and stable systems that ensure the reliability of the three measurements (see the cyclic voltammetry (CV) in Figure S4 in the Supporting Information). The low-frequency limit used was $0.1 \mathrm{~Hz}$ because no more features were observed below this frequency. On the other hand, the high frequencies were instrumentally limited, reaching 310, 200, and $16 \mathrm{kHz}$ for IS, IMPS, and IMVS, respectively. The measurements were performed at open-circuit, the most common condition for IMVS, very typical for IS and recently, also used for IMPS..$^{[7,27,30]}$ In the Experimental Section, we provide further details of the experimental procedures.

We first verified that our experimental data satisfied Equation (4) by calculating the quotient of $W$ and $Q$ for each frequency, see red triangles in Figure 4a. A very good match between the measured IS spectrum and the one obtained from IMPS and IMVS is observed.

\subsubsection{Selection of the Equivalent Circuit}

The IS spectrum of the silicon photodiode (Figure 4a) presents one large arc at low frequencies and a secondary process at high frequencies. In the IMPS spectrum (Figure 4c), we also observe two processes, although their characteristic frequencies appear closer than in the IS spectrum, resulting in more distorted arcs. In contrast, the IMVS spectrum (Figure 4d) shows only one large arc. However, as we mentioned above, IMVS could only be measured up to $16 \mathrm{kHz}$, so we speculate that one process is likely hidden in the IMVS spectrum at higher frequencies. The fact that the projection of the IMVS arc does not end at the origin of the $W^{\prime}$ and $W^{\prime \prime}$ axes supports this assumption.

To analyze the obtained spectra with the developed theory, we must first reduce the GEC in Figure $3 a$ to a particular case, replacing the general impedances $\left(Z_{\mathrm{S}}, Z_{\mathrm{rec}}, Z_{\mathrm{p}}\right.$, and $\left.Z_{\mathrm{f}}\right)$ by specific elements such as resistances and capacitances. This choice is complicated by the fact that different circuits can reproduce the general spectral features of combinations of any two of IS, IMPS, and IMVS. ${ }^{40]}$ This is shown in Figure 5 for fitting of the experimental IS spectra and simulations with the corresponding fitted parameters of IMPS and IMVS spectra using different ECs. For example, an $R \| C$ element in both $Z_{\mathrm{f}}$ (Figure 5e) and $Z_{\mathrm{S}}$ (Figure $5 \mathrm{f}$ ) gives an arc in the lower quadrant of the IMPS spectrum (Figure 5b) while yielding two arcs also in the impedance spectrum (Figure 5a). However, when also considering the IMVS response (Figure $5 \mathrm{~d}$ ), we observe a difference in the generated spectra. We therefore use this logic to compare the response of different ECs with that of the experimental spectra of the three techniques to narrow down the number of ECs available for fitting the spectra. A detailed discussion of the different equivalent circuits in Figure 5 is provided in Section S2.1 of the Supporting Information. As an example of the method, we note that the ECs in Figure $5 \mathrm{e}-\mathrm{g}$ have an $R \| C$ pair placed in $Z_{\mathrm{f}}, Z_{\mathrm{S}}$, and $Z_{\text {rec }}$, respectively. The ECs in Figure 5 e,f can be easily discarded because they present an arc in the bottom quadrant of the IMPS spectra (red and green lines, respectively, in Figure 5b) which is not present in the experimental spectrum. On the other hand, the IMPS and IMVS simulations corresponding to the circuit in Figure $5 \mathrm{~g}$ (purple lines in Figure $5 \mathrm{~b}-\mathrm{d}$ ) reach negative values of the real components, which are also not present in the experimental spectrum.

The selected EC as a consequence of this procedure is shown in Figure $5 f$, where $Z_{\mathrm{S}}=R_{\mathrm{S}}, Z_{\mathrm{f}}=0$, and $Z_{\text {rec }}^{\mathrm{T}}$ is given by the combination of $R_{\mathrm{rec}}, R_{1}, C_{1}$, and $C_{2}$, which reproduce the three spectra simultaneously (see blue lines in Figure 5 ). The condition $Z_{\mathrm{f}}=0$ (discussed in Section 2.1.3) allows estimating $Z_{\mathrm{S}}$ and $Z_{\text {rec }}^{\mathrm{T}}$ through Equations (18) and (19). The $Z_{\mathrm{S}}$ spectra 

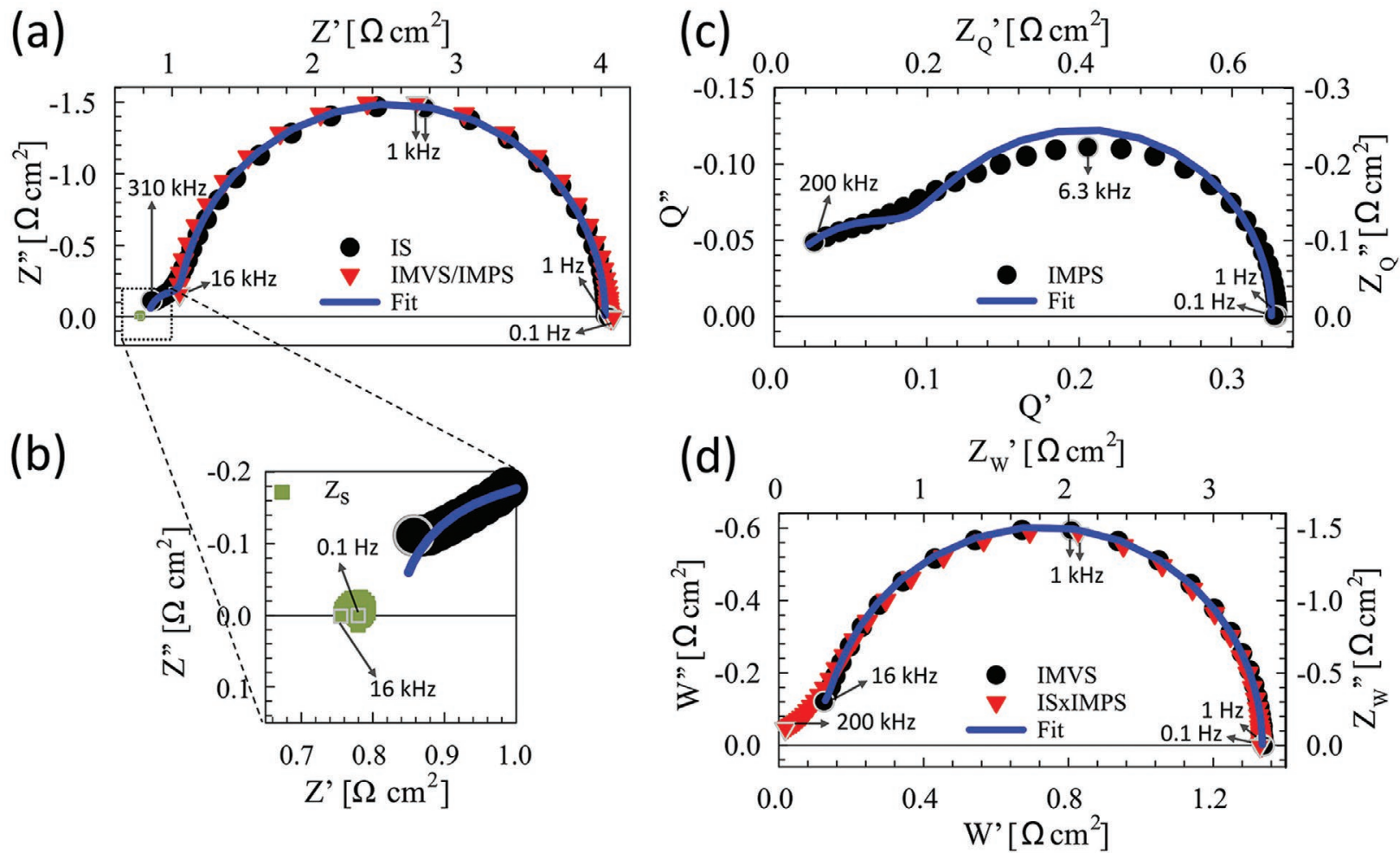

Figure 4. Black dots represent measured a,b) IS, c) IMPS, and d) IMVS spectra of a silicon photodiode, under open-circuit conditions and $89 \mathrm{~mW} \mathrm{~cm}^{-2}$ blue LED illumination. Red triangles represent the quotient between IMVS and IMPS in (a) and the product between IS and IMPS in (d). The top and right axis of (c) and (d) represents $Z_{Q}=Q R_{S} / \eta_{\text {a.s }}$ and $Z_{W}=W / \eta_{\text {a.s }}$, respectively, as given by Equations (29) and (30). The blue lines are the fittings of $a, b)$ IS, c) IMPS, and d) IMVS employing the simplified GEC in Figure $5 \mathrm{~h}$. The green squares in (b), inset of (a), is the series impedance $\left(Z_{S}\right)$ obtained from the combination of IS with IMVS through Equation (19).

calculated by combining IS and IMVS data can be seen (green squares) in Figure 4a and, with more detail, in its inset plot in Figure 4b. It approaches very well to a single point on the real axis, the expected behavior of a resistor $\left(R_{\mathrm{S}}\right)$. This yields the rest of the impedance (the two arcs in Figure 4a) to $Z_{\text {rec }}^{\mathrm{T}}$. We clarify that the chosen $\mathrm{EC}$ is not a unique equivalent circuit that fits all three spectra, an example of an alternative is shown in Section S2.2 in the Supporting Information. Our procedure serves to reduce the large number of EC options available for fitting by using the experimental spectra as a quality check. While providing general rules for identifying the correct EC is an attractive proposition, it is a very difficult goal due to the generality of the model, whose elements can be arbitrary combinations of passive elements. In addition, we note that the structure of the device must also be taken into consideration when choosing an equivalent circuit for devices that contain multiple layers in addition to that of the absorber, such as PSCs that employ selective contact layers. The generality of our developed model allows the inclusion of passive elements in different parts of the EC to easily model these additional layers in the device.

\subsubsection{Fitting Results}

As mentioned in the introduction, analysis of IMPS or IMVS beyond the calculation of characteristic times has been very uncommon until now. ${ }^{[30,34]}$ To the best of our knowledge, the fitting of IMPS and IMVS spectra with an equivalent circuit, in a similar way to what is done conventionally for IS spectra, has not been done previously. The main reason for this fact was the absence of a model that connected the IMPS or IMVS transfer functions to the electrical elements such as resistances and capacitances that are used for the analysis of the impedance. Here, the connection developed in the theoretical framework (Section 2.1) through $\eta_{\text {a.s }}$ allows simultaneous fitting with the three techniques, after discarding (using our selection procedure) many potential equivalent circuits that do not provide compatible results. We show the potential of this method in the analysis of the particular case of a silicon photodiode through the EC in Figure $5 \mathrm{~h}$.

Provided the condition $Z_{\mathrm{f}}=0$ given by the photodiode and considering the EC of Figure $5 \mathrm{~h}$, the transfer functions in Equations (11)-(13) reduce to

$Z=R_{\mathrm{S}}+Z_{\text {rec }}^{\mathrm{T}}=R_{\mathrm{S}}+\left(\frac{1}{R_{r e c}}+\frac{1}{R_{1}+\left(i \omega C_{1}\right)^{-1}}+i \omega C_{2}\right)^{-1}$

$Q=\frac{\eta_{\text {a:s }}}{R_{\mathrm{S}}}\left(\frac{1}{R_{\mathrm{S}}}+\frac{1}{Z_{\text {rec }}^{\mathrm{T}}}\right)^{-1}=\frac{\eta_{\mathrm{a} \cdot \mathrm{s}}}{R_{\mathrm{S}}}\left(\frac{1}{R_{\mathrm{S}}}+\frac{1}{R_{\text {rec }}}+\frac{1}{R_{1}+\left(i \omega C_{1}\right)^{-1}}+i \omega C_{2}\right)^{-1}$

$\mathrm{W}=\eta_{\mathrm{a} \cdot \mathrm{s}} Z_{\text {rec }}^{\mathrm{T}}=\eta_{\mathrm{a} \cdot \mathrm{s}}\left(\frac{1}{R_{\text {rec }}}+\frac{1}{R_{1}+\left(i \omega C_{1}\right)^{-1}}+i \omega C_{2}\right)^{-1}$ 
(a)

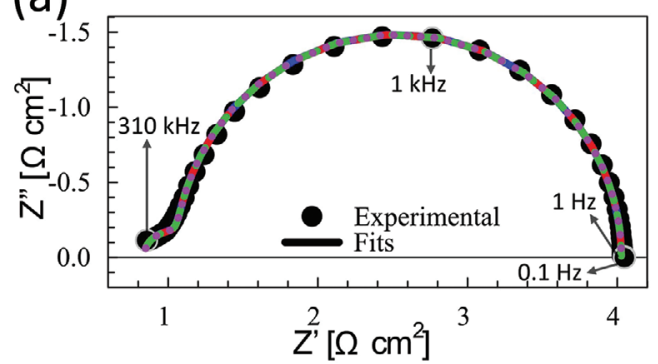

(b)

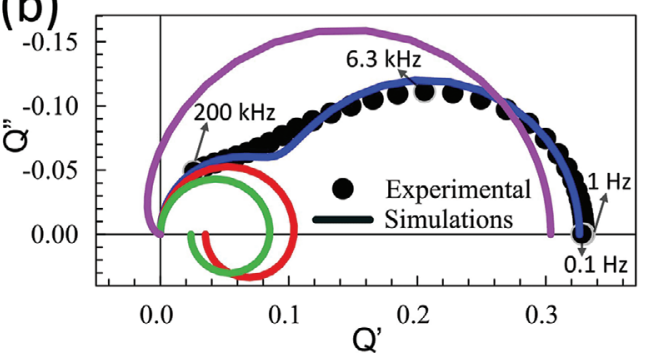

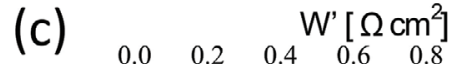

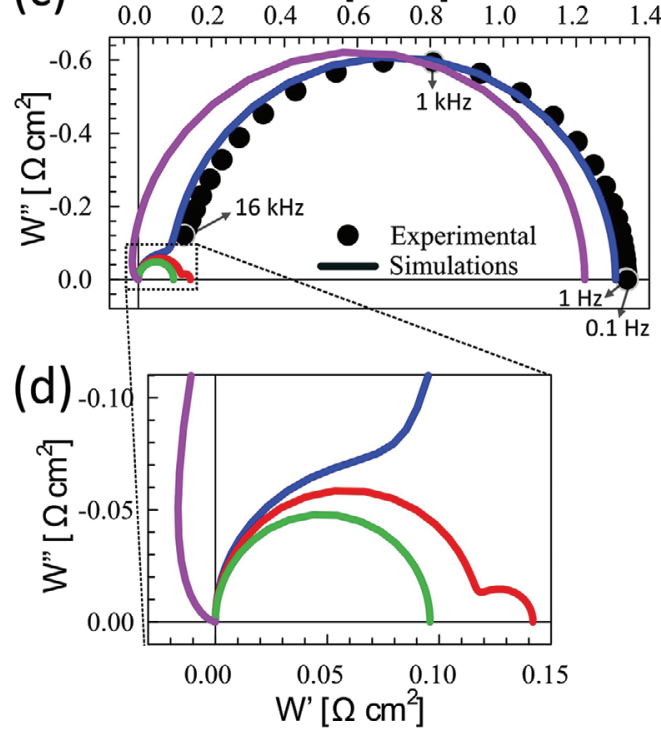

(e) EC1 $\xi_{\phi}$

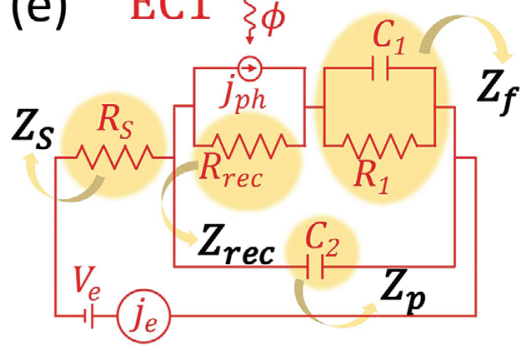

(f) $\mathrm{EC} 2$

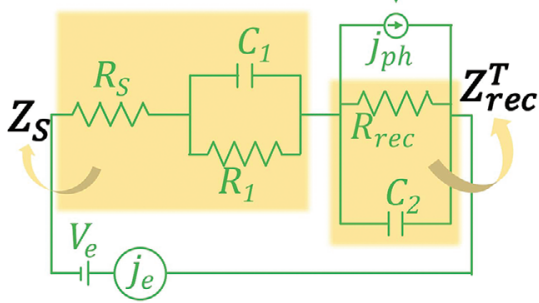

(g) $\mathrm{EC} 3 \xi$
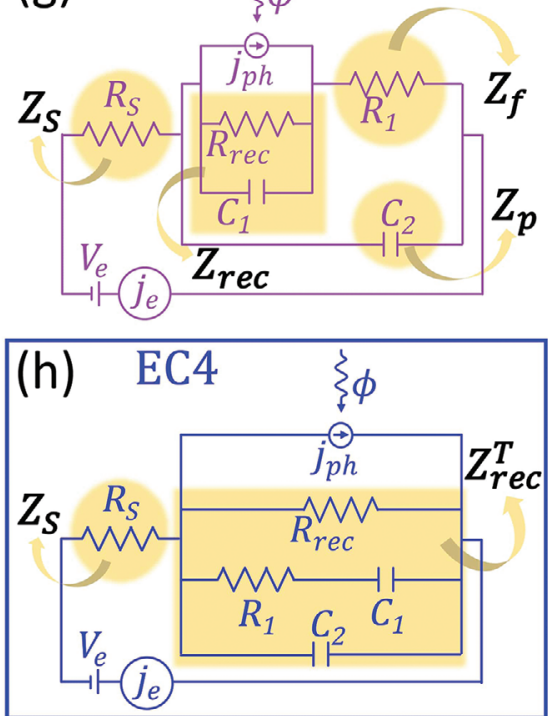

Figure 5. Black dots show measured a) IS, b) IMPS, and c) IMVS for a silicon photodiode, under OC condition and $89 \mathrm{~mW} \mathrm{~cm}^{-2}$ blue light (470 nm peak), also shown in Figure 4 The different lines in (a) are the resulting fits employing the equivalent circuits in (e), (f), (g), and (h), respectively with the color. Following the same color pattern, the lines in (b), (c), and (d) are the IMPS and IMVS simulations corresponding to the IS fitting results and considering $\eta_{\text {a.s }}$ in Table 1.

IS data were fitted using standard software for IS analysis (ZView in our case) using the equivalent circuit plot in Figure $5 \mathrm{~h}$, reduced to Figure $\mathrm{S} 3 \mathrm{e}$ in the Supporting Information. Results of the fitting of these data are plotted as a blue curve in Figure 4a and the parameters obtained are shown in the "IS" column of Table 1.

Equation (21) shows that the transfer function of IMPS is simply the impedance of the parallel combination of $R_{\mathrm{S}}$ and $Z_{\text {rec }}^{\mathrm{T}}$ multiplied by a constant $\eta_{\mathrm{a} \cdot \mathrm{s}} / R_{\mathrm{S}}$. Similarly, the transfer function of IMVS written in Equation (22) is just the impedance $Z_{\text {rec }}^{\mathrm{T}}$ multiplied by $\eta_{\mathrm{a} . \mathrm{s}}$. Therefore, the same tools used to fit IS serve to fit the IMPS and IMVS spectra. To apply this analysis procedure, Equations (21) and (22) may be rewritten as

$Q=\left[\frac{1}{R_{r e c}^{Q}}+\frac{1}{R_{1}^{Q}+\left(i \omega C_{1}^{Q}\right)^{-1}}+i \omega C_{2}^{Q}\right]^{-1}$

and

$W=\left[\frac{1}{R_{r e c}^{W}}+\frac{1}{R_{1}^{W}+\left(i \omega C_{1}^{W}\right)^{-1}}+i \omega C_{2}^{W}\right]^{-1}$ 
Table 1. Parameters obtained from the fitting of IS, IMPS, and IMVS data in Figure 4 with the equivalent circuit in Figure $5 \mathrm{~h}$. The "IS" column corresponds to the direct fitting of impedance spectroscopy data in Figure $4 a$ to the equivalent impedance shown in Figure S3e in the Supporting Information. The "IMPS+IMVS" column corresponds to the parameters obtained by combining the fittings of IMPS and IMVS with the ECs adapted for these techniques in Figure S3g,i in the Supporting Information, respectively.

\begin{tabular}{lccc}
\hline & IS & IMPS+IMVS & Direct measurements \\
\hline$R_{\mathrm{S}}\left[\Omega \mathrm{cm}^{2}\right]$ & $0.82 \pm 0.03$ & $0.80 \pm 0.06$ & - \\
$R_{\mathrm{rec}}\left[\Omega \mathrm{cm}^{2}\right]$ & $3.19 \pm 0.01$ & $3.2 \pm 0.2$ & - \\
$C_{2}\left[\mu \mathrm{H} \mathrm{cm}^{-2}\right]$ & $8.0 \pm 0.4$ & $8.0 \pm 0.7$ & - \\
$R_{1}\left[\Omega \mathrm{cm}^{2}\right]$ & $0.37 \pm 0.01$ & $0.42 \pm 0.05$ & - \\
$C_{1}\left[\mu \mathrm{F} \mathrm{cm}^{-2}\right]$ & $31.1 \pm 0.4$ & $28 \pm 4$ & - \\
$R_{\mathrm{DC}}\left[\Omega \mathrm{cm}^{2}\right]$ & $4.01 \pm 0.03$ & $4.0 \pm 0.2$ & $4.1 \pm 0.2^{\mathrm{a})}$ \\
$\tau_{\mathrm{LF}}^{l S}=\tau_{\mathrm{LF}}^{\mid \mathrm{MVS}}[\mathrm{ms}]$ & $125 \pm 2$ & $116 \pm 16$ & $126 \pm 24$ \\
$\tau_{\mathrm{LF}}^{I M P S}[\mathrm{~ms}]$ & $25.5 \pm 0.7$ & $23 \pm 3$ & $25 \pm 5$ \\
$a[\%]$ & - & - & $43 \pm 2^{\mathrm{b})}$ \\
$\eta_{\text {a:s }}[\%]$ & - & $41 \pm 3$ & - \\
$\eta_{\text {col-diff }}[\%]$ & $79.6 \pm 0.7$ & $80 \pm 7$ & - \\
$\eta_{\text {sep }}[\%]$ & - & $95 \pm 8$ & - \\
\hline
\end{tabular}

a) The $R_{\mathrm{DC}}$ was obtained from the inverse of the slope of the current-voltage curve at the $V_{\mathrm{OC}}$ (see Figure S4 in the Supporting Information); b) The absorptance $(a)$ was obtained from reflectance measurements.

where the parameters with the superscripts $Q$ and $W$ can now be obtained directly from the fitting (see Figure S3f,h, Supporting Information). These parameters are related to the resistances and capacitances in the EC of Figure $5 \mathrm{~h}$ (obtained from IS) through

$\frac{\eta_{\mathrm{a} \cdot \mathrm{s}}}{R_{\mathrm{S}}}=\frac{C_{1}}{C_{1}^{Q}}=\frac{C_{2}}{C_{2}^{Q}}=\frac{R_{1}^{Q}}{R_{1}}=R_{\text {rec }}^{Q}\left(\frac{1}{R_{\mathrm{S}}}+\frac{1}{R_{\text {rec }}}\right)$

and

$\eta_{\text {a:s }}=\frac{C_{1}}{C_{1}^{W}}=\frac{C_{2}}{C_{2}^{W}}=\frac{R_{1}^{W}}{R_{1}}=\frac{R_{r e c}^{W}}{R_{r e c}}$

Similarly, the relation between the parameters obtained from IMPS and IMVS is given by

$R_{\mathrm{S}}=\frac{C_{1}^{Q}}{C_{1}^{W}}=\frac{C_{2}^{Q}}{C_{2}^{W}}=\frac{R_{1}^{W}}{R_{1}^{Q}}$

and

$\eta_{\text {a:s }}=\left(\frac{1}{R_{\text {rec }}^{Q}}-\frac{R_{\mathrm{S}}}{R_{\text {rec }}^{W}}\right)^{-1}$

Therefore, $\eta_{\text {a.s }}$ and $R_{\mathrm{S}}$ can be obtained through the combination of any two of these techniques. The results of the IMPS and IMVS fits are represented in blue lines in Figure 4c,d, with the EC parameters and $\eta_{\text {a.s }}$ obtained from Equations (25)-(28) in the "IMPS+IMVS" column of Table 1. Before discussion of the parameters, we introduce an alternate method of fitting the IMPS and IMVS spectra. We define pseudo-impedances of IMPS and IMVS as

$$
\begin{aligned}
& Z_{Q}=Q \frac{R_{\mathrm{S}}}{\eta_{\mathrm{a} \cdot \mathrm{s}}} \\
& Z_{W}=\frac{W}{\eta_{\mathrm{a} \cdot \mathrm{s}}}
\end{aligned}
$$

Using these impedances, we transformed the experimental spectra to a pseudo-impedance form, see top and right axis of Figure 4c,d. With these last transformations, the fitting of the data, through the EC in Figure S3g,i in the Supporting Information (see Section S3.2 in the Supporting Information), provides the same parameters as those obtained through IS, as can be seen in " $Z_{Q}$ " and " $Z_{W}$ " columns of Table S1 in the Supporting Information. These fits are plotted as the blue lines in Figure 4c,d.

With the results of the fitting, the low-frequency characteristic times of each technique can be calculated. The derivation of the mathematical expressions is detailed in Section S4 of the Supporting Information, the results are shown in Equations (S50), (S52), and (S55) in the Supporting Information, and calculated in Table 1 and Table S1 in the Supporting Information. Note that the low-frequency characteristic time of IS is the same as that of IMVS, but different from that of IMPS. These characteristic times were also obtained directly from the measured spectra (see Figure $4 a, c, d$ and last column of Table 1).

Once all the elements of the EC in Figure $5 \mathrm{~h}$ were obtained, it was possible to calculate further useful parameters, presented in Table 1 and Table S1 in the Supporting Information. The DC resistance $\left(R_{\mathrm{DC}}\right)$ and collection efficiency $\left(\eta_{\text {col-diff }}\right)$ were calculated from Equation (S20) and (S23) in the Supporting Information, respectively. The differential external quantum efficiency $\mathrm{EQE}_{\mathrm{PV} \text {-diff }}$ was calculated from Equation (S15) in the Supporting Information as can be seen in columns " $Z_{Q}$ " and "IMPS+IMVS" of Table S1 in the Supporting Information, an alternative way from the low-frequency limit of the IMPS (see Figure 3c). ${ }^{[40]}$ By directly measuring the absorptance (a) (last column of Table 1), it was possible to calculate $\eta_{\text {sep }}$

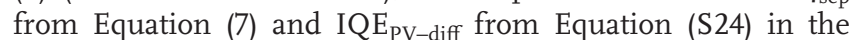
Supporting Information. For the silicon photodiode, $\eta_{\text {sep }}$ is very close to 1 (indistinguishable, considering the error interval), which means that, as expected for this device, ${ }^{[35,36]}$ the approximation of $\eta_{\mathrm{a} \cdot \mathrm{s}} \approx a$ is very reasonable.

All sets of results in the different columns of Table 1 and in Table S1 in the Supporting Information match very well, including those obtained from direct measurements with independent techniques. This match confirms our main assumption that the three spectra (IS, IMPS, and IMVS) in Figure 4 correspond to the same internal process of the device. Second, it proves that we can model the three techniques with a simple equivalent circuit (i.e., Figure 5h). And last but not least, it validates the procedures developed to obtain the characteristic parameters of the device, such as time constants, capacitances, and resistances. Furthermore, by combining any of these two techniques, these procedures allow calculating optoelectronic parameters such $\eta_{\mathrm{a} \cdot \mathrm{s}}, \eta_{\text {col-diff, }} \mathrm{EQE}_{\mathrm{PV} \text {-diff, }}$ and together with absorptance measurements, $\eta_{\mathrm{sep}}$, and $\mathrm{IQE}_{\mathrm{PV} \text {-diff. }}$ 


\section{Conclusions}

This work provides a strong framework for the analysis of solar cells and photoelectrochemical devices by combining different small-perturbation frequency-domain techniques, namely, IS, IMPS, and IMVS. First, we have shown that the three techniques are properly correlated only when accounting for the absorptance and separation efficiency. Using this relationship, we have developed a generalized equivalent circuit and its associated theoretical framework that can be used to model the response of all three techniques together. The potential of this tool was demonstrated experimentally for a silicon photodiode. Combining the experimental results of the three techniques, the selection of the equivalent circuit was narrowed down, leading to an equivalent circuit that accurately models all three experimental responses simultaneously. Additionally, we have developed a method to fit the three spectra with standard software for impedance analysis using the identified equivalent circuit, allowing to obtain high quality and reliability of the output parameters from the model. Furthermore, combining these techniques in pairs, electro-optical parameters such as the separation efficiency, together with differential external and internal quantum efficiencies were obtained that are inaccessible from individual use of any of the three techniques. The generality of the developed procedure allows easy extension to the analysis of other photosensitive devices.

\section{Experimental Section}

The IS, IMPS, and IMVS measurements were performed on a commercial silicon photodiode (SiPh, Thorlabs FDS100). IS, IMPS, IMVS, and CV were measured with an Autolab PGSTAT302 equipped with an FRA32M module and combined with the light-emitting diode (LED) driver. The four techniques were performed under $89 \mathrm{~mW} \mathrm{~cm}$-2 light intensity generated by an array of three blue LEDs (Philips LUMILEDS LXML-PB01-0040 with $470 \mathrm{~nm}$ peak). The $\mathrm{CV}$ was performed at $50 \mathrm{mV} \mathrm{s}^{-1}$. For the IS measurement, a $20 \mathrm{mV}$ AC perturbation was applied. For IMPS and IMVS, an AC perturbation equal to $10 \%$ of the light flux was applied. The low-frequency limit was $0.1 \mathrm{~Hz}$ for the three techniques and the high frequencies were instrumentally limited, reaching 310, 200, and $16 \mathrm{kHz}$ for IS, IMPS, and IMVS, respectively. The measurements were performed at open-circuit. Analysis of all spectra was done using Zview software.

\section{Supporting Information}

Supporting Information is available from the Wiley Online Library or from the author.

\section{Acknowledgements}

This project has received funding from the European Union's Horizon 2020 MSCA Innovative Training Network under grant agreement no. 764787. F.F.-S. acknowledges Ministerio de Economía y Competitividad (MINECO) from Spain under the project ENE2017-85087-C3-7-R and Generalitat Valenciana under the project PROMETEO/2020/028 for financial support. S.R. acknowledges funding from the Helmholtz association via the project PEROSEED.

\section{Conflict of Interest}

The authors declare no conflict of interest.

\section{Data Availability Statement}

The data that support the findings of this study are openly available in http://hdl.handle.net/10234/193441.

\section{Keywords}

charge separation efficiency, impedance spectroscopy, IMPS, IMVS, photodiodes

Received: June 21, 2021

Revised: July 30, 2021

Published online:

[1] L. Bertoluzzi, J. Bisquert, J. Phys. Chem. Lett. 2017, 8, 172.

[2] D. Klotz, D. S. Ellis, H. Dotan, A. Rothschild, Phys. Chem. Chem. Phys. 2016, 18, 23438.

[3] L. M. Peter, Chem. Rev. 1990, 90, 753.

[4] J. Li, R. Peat, L. Peter, J. Electroanal. Chem. Interfacial Electrochem. 1984, 165, 41.

[5] P. De Jongh, D. Vanmaekelbergh, Phys. Rev. Lett. 1996, 77, 3427.

[6] J. Krüger, R. Plass, M. Grätzel, P. J. Cameron, L. M. Peter, J. Phys. Chem. B 2003, 107, 7536.

[7] S. Ravishankar, A. Riquelme, S. K. Sarkar, M. Garcia-Batlle, G. Garcia-Belmonte, J. Bisquert, J. Phys. Chem. C 2019, 123, 24995.

[8] A. Pockett, M. Spence, S. K. Thomas, D. Raptis, T. Watson, M. J. Carnie, Sol. RRL 2021, 5, 2100159.

[9] G. Schlichthörl, S. Y. Huang, J. Sprague, A. J. Frank, J. Phys. Chem. B 1997, 101, 8141.

[10] R. Kern, R. Sastrawan, J. Ferber, R. Stangl, J. Luther, Electrochim. Acta 2002, 47, 4213.

[11] F. Fabregat-Santiago, E. M. Barea, S. Giménez, J. Bisquert, in Molecular Devices for Solar Energy Conversion and Storage, (Eds: H. Tian, G. Boschloo, A. Hagfeldt), Springer Singapore, Singapore 2018, p. 353.

[12] J. Bisquert, F. Fabregat-Santiago, in Dye-Sensitized Solar Cells, (Ed: K. Kalyanasundaram), CRC Press, Switzerland 2010, p. 457.

[13] L. Dloczik, O. Ileperuma, I. Lauermann, L. Peter, E. Ponomarev, G. Redmond, N. Shaw, I. Uhlendorf, J. Phys. Chem. B 1997, 101, 10281.

[14] J. Bisquert, V. S. Vikhrenko, J. Phys. Chem. B 2004, 108, 2313.

[15] H. Cachet, E. M. M. Sutter, J. Phys. Chem. C 2015, 119, 25548.

[16] J. E. Thorne, J.-W. Jang, E. Y. Liu, D. Wang, Chem. Sci. 2016, 7, 3347.

[17] D. Cardenas-Morcoso, A. Bou, S. Ravishankar, M. García-Tecedor, S. Gimenez, J. Bisquert, ACS Energy Lett. 2019, 5, 187.

[18] A. Todinova, J. s. Idígoras, M. Salado, S. Kazim, J. A. Anta, J. Phys. Chem. Lett. 2015, 6, 3923.

[19] L. Contreras, J. Idígoras, A. Todinova, M. Salado, S. Kazim, S. Ahmad, J. A. Anta, Phys. Chem. Chem. Phys. 2016, 18, 31033.

[20] E. Ponomarev, L. Peter, J. Electroanal. Chem. 1995, 396, 219.

[21] L. M. Peter, J. Solid State Electrochem. 2013, 17, 315.

[22] A. Riquelme, F. E. Gálvez, L. Contreras-Bernal, H. Míguez, J. A. Anta, J. Appl. Phys. 2020, 128, 133103.

[23] J. Bisquert, S. Giménez, L. Bertoluzzi, I. Herraiz-Cardona, in Photoelectrochemical Solar Fuel Production, (Eds: S. Giménez, J. Bisquert), Springer, Cham 2016, p. 281. 
[24] V. Babu, R. F. Pineda, T. Ahmad, A. O. Alvarez, L. A. Castriotta, A. D Carlo, F. Fabregat-Santiago, K. Wojciechowski, ACS Appl. Energy Mater. 2020, 3, 5126.

[25] A. O. Alvarez, R. Arcas, C. A. Aranda, L. Bethencourt, E. MasMarzá, M. Saliba, F. Fabregat-Santiago, J. Phys. Chem. Lett. 2020, 11, 8417.

[26] O. Almora, C. Aranda, G. Garcia-Belmonte, J. Phys. Chem. C 2018, $122,13450$.

[27] J. Halme, Phys. Chem. Chem. Phys. 2011, 13, 12435.

[28] J. Bisquert, M. Janssen, J. Phys. Chem. Lett. 2021, 12, 7964.

[29] A. Bou, A. Pockett, D. Raptis, T. Watson, M. J. Carnie, J. Bisquert, J. Phys. Chem. Lett. 2020, 11, 8654.

[30] O. Almora, Y. Zhao, X. Du, T. Heumueller, G. J. Matt, G. Garcia-Belmonte, C. J. Brabec, Nano Energy 2020, 75, 104982.

[31] O. Almora, D. Miravet, G. J. Matt, G. Garcia-Belmonte, C. J. Brabec, Appl. Phys. Lett. 2020, 116, 013901.
[32] S. Ravishankar, M. Garcia-Batlle, J. Bisquert, G. Garcia-Belmonte, J. Odrobina, C.-A. Schiller, J. Phys. Chem. C 2020, 124, 15793.

[33] G. Tumen-Ulzii, T. Matsushima, D. Klotz, M. R. Leyden, P. Wang, C. Qin, J.-W. Lee, S.-J. Lee, Y. Yang, C. Adachi, Commun. Mater. 2020, 1, 31

[34] S. Ravishankar, C. Aranda, S. Sanchez, J. Bisquert, M. Saliba, G. Garcia-Belmonte, J. Phys. Chem. C 2019, 123, 6444.

[35] J. Bisquert, The Physics of Solar Cells: Perouskites, Organics, and Photovoltaic Fundamentals, CRC Press, Boca Raton, FL 2017.

[36] T. Kirchartz, J. Bisquert, I. Mora-Sero, G. Garcia-Belmonte, Phys. Chem. Chem. Phys. 2015, 17, 4007.

[37] J. Bisquert, ACS Energy Lett. 2010, 646, 43.

[38] J. Bisquert, Phys. Chem. Chem. Phys. 2003, 5, 5360.

[39] S. R. Raga, E. M. Barea, F. Fabregat-Santiago, J. Phys. Chem. Lett. 2012, 3, 1629.

[40] S. Fletcher, J. Electrochem. Soc. 1994, 141, 1823. 